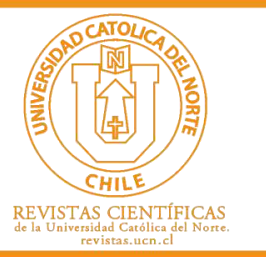

\title{
Molecular descriptors of certain OTIS interconnection networks
}

\author{
Murat Cancan ${ }^{1}$ (10 orcid.org/0000-0002-8606-2274 \\ Iftikhar Ahmad ${ }^{2}$ (1) orcid.org/0000-0001-5879-5801 \\ Sarfarz Ahmad ${ }^{3}$
}

${ }^{1}$ Van Yznc Yil University, Faculty of Education, Van, Turkey.

m_cencen@yahoo.com

${ }^{2}$ COMSATS University Islamabad, Dept. of Mathematics, Lahore, Pakistan.

活fi6301@gmail.com

${ }^{3}$ Riphah International University, Dept. of Mathematics, Lahore, Pakistan.

sarfrazahmad@cuilahore.edu.pk

Received: February 2020 | Accepted: May 2020

\begin{abstract}
:
Network theory as an important role in the field of electronic and electrical engineering, for example, in signal processing, networking, communication theory, etc. The branch of mathematics known as Graph theory found remarkable applications in this area of study. A topological index (TI) is a real number attached with graph networks and correlates the chemical networks with many physical and chemical properties and chemical reactivity. The Optical Transpose Interconnection System (OTIS) network has received considerable attention in recent years and has a special place among real world architectures for parallel and distributed systems. In this report, we compute redefined first, second and third Zagreb indices of OTIS swapped and OTIS biswapped networks. We also compute some Zagreb polynomials of understudy Networks.
\end{abstract}

Keywords: Zagreb index; Zagreb polynomial; Networks; Chemical graph theory.

MSC (2020): 05C15, 05C38, 05C40.

\section{Cite this article as (IEEE citation style):}

M. Cancan, I. Ahmad, and S. Ahmad, "Molecular descriptors of certain OTIS interconnection networks", Proyecciones (Antofagasta, On line), vol. 39, no. 4, pp. 769-786, Aug. 2020, doi: 10.22199/issn.0717-6279-2020-04-0048.

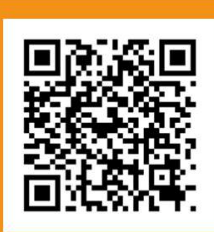

Article copyright: (c) 2020 Murat Cancan, Iftikhar Ahmad, and Sarfarz Ahmad. This is an open access article distributed under the terms of the Creative Commons License, which permits unrestricted use and distribution provided the original author and source are credited. 


\section{Introduction}

Mathematical chemistry is an area of research in chemistry in which mathematical tools are used to solve problems of chemistry. Chemical graph theory is an important area of research in mathematically chemistry which deals with topology of molecular structure such as the mathematical study of isomerism and the development of topological descriptors or indices. Infect, TIs are real numbers attached with graph networks and graph of chemical compounds and has applications in quantitative structure-property relationships. TIs remain invariant upto graph isomorphism and help to predict many properties of chemical compounds, networks and nanomaterials, for example, viscosity, boiling points, radius of gyrations, etc without going to lab $[3,5,24]$.

Other emerging field is Cheminformatics, in which we use QSAR and QSPR relationship to guess biological activity and chemical properties of nanomaterial and networks. In these investigations, some Physico-chemical properties and TIs are utilized to guess the behavior of chemical networks [11]. Like TIs, polynomials also fund considerable applications in network theory and chemistry, for example, Hosoya polynomial, which is also known as Wiener polynomial, introduced in [13] plays an important role in computation of distance-based TIs. M-polynomial [8] was defined in 2015 and plays a similar role in computation of numerous degree-based TIs $[1,16,17,18,19]$. The M-polynomial contains precious information about degree-based TIs and many TIs can be computed from this simple algebraic polynomial. The first TI was defined in 1947 by Weiner during studying boiling point of alkanes [26]. This index is now known as Weiner index. Thus Weiner established the framework of TIs and the Wiener index is initially the first and most concentrated TI. For details about applications of graph theory and TIs, see $[12,15,23,25]$ and reference therein.

The other oldest TI is Randić index (RI), given by Milan Randic [20] in 1975. After the success of Randić index, in the year 1988, the generalized version of Randić index was introduced [4,7]. This version attracts both the mathematicians and chemists $[2,19]$.

The RI is a most mainstream regularly connected and most concentrated among all other TIs. Numerous research papers and text books are published in different academic journals on this TI. Two surveys on RI was written by Milan Randić and the reason behind the success of such a simple $\mathrm{TI}$ is as yet a puzzle, although some conceivable clarifications were given.

After Randić index, the most studied TIs are 1st Zagreb index (ZI) and 
2nd ZI [14]. In this report we aim to study some Zagreb polynomials and redefined Zagreb indices of OTIS (swapped and biswapped) networks.

\section{Basic Notions}

In mathematical chemistry, precisely speaking, in chemical-graph-theory (CGT), a molecular graph and graph network is a simple and connected graph, in which atoms represents vertices and chemical bonds represents edges. We reserve $\mathrm{G}$ for simple connected graph, $\mathrm{E}$ for edge set and $\mathrm{V}$ for vertex set throughout the thesis. The degree of a vertex $u$ of graph $\mathrm{G}$ is the number of vertices that are attached with $\mathrm{u}$ and is denoted by $d_{v}$. With the help of TIs, many properties of molecular structure can be obtained without going to lab. The reality is, many research paper has been written on computation of degree-based indices and polynomials of different molecular structure and networks but only few work has been done so far on distance based indices and polynomials. Our aim is to compute distancebased as well as degree-based indices of understudy networks. The first and the second ZIs (cf. [14]) are defined as

$$
M_{1}(G)=\sum_{u v \in E(G)}\left(d_{u}+d_{v}\right)
$$

and

$$
M_{2}(G)=\sum_{u v \in E(G)}\left(d_{u} \times d_{v}\right) .
$$

Considering the ZIs, Fath-Tabar in [10] introduced the following first and the second Zagreb polynomials

$$
M_{1}(G, x)=\sum_{u v \in E(G)} x^{d_{u}+d_{v}}
$$

and

$$
M_{2}(G, x)=\sum_{u v \in E(G)} x^{d_{u} \times d_{v}} .
$$

The properties of first and second Zagreb polynomials for some chemical structures have been studied in the literature [21].

After the success of ZIs, the researchers in [9], introduced the following third ZI

$$
M_{3}(G)=\sum_{u v \in E(G)}\left|d_{u}-d_{v}\right|
$$


and the third Zagreb polynomial

$$
M_{3}(G, x)=\sum_{u v \in E(G)} x^{\left|d_{u}+d_{v}\right|} .
$$

The other Zagreb type polynomials are introduced in [6] in 2016

$$
\begin{aligned}
M_{4}(G, x) & =\sum_{u v \in E(G)} x^{d_{u}\left(d_{u}+d_{v}\right)}, \\
M_{5}(G, x) & =\sum_{u v \in E(G)} x^{d_{v}\left(d_{u}+d_{v}\right)}, \\
M_{a, b}(G, x) & =\sum_{u v \in E(G)} x^{a d_{u}+b d_{v}}, \\
M_{a, b}^{\prime}(G, x) & =\sum_{u v \in E(G)} x^{\left(d_{u}+a\right)\left(d_{v}+b\right)} .
\end{aligned}
$$

Redefined ZIs are defined in [22] by Ranjini et al.

$$
\begin{gathered}
\operatorname{Re} Z G_{1}(G)=\sum_{u v \in E(G)} \frac{d_{u}+d_{v}}{d_{u} d_{v}}, \\
\operatorname{Re} Z G_{2}(G)=\sum_{u v \in E(G)} \frac{d_{u} d_{v}}{d_{u}+d_{v}}, \\
\operatorname{Re} Z G_{3}(G)=\sum_{u v \in E(G)}\left(d_{u}+d_{v}\right)\left(d_{u} d_{v}\right) .
\end{gathered}
$$

\section{Methodology}

There are three kinds of invariants:

1) Degree-based TIs

2) Distance-based TIs

3) Spectral-based TIs

In this thesis, we focus on degree-based and distance-based graph invariants. To compute degree-based invariants, we divide the edge set of graph networks into classes based on the degree of the end vertices and compute there cardinality. From this edge partition, we compute our desired results.

\section{Computational results}

Now we give our main results. 


\subsection{Topological indices of $O R_{k}$ OTIS swapped networks}

Let $R_{k}$ be k-regular graph on n vertices and $O R_{k}$ be OTIS swapped network with basis network $R_{k}$. Figure 1 depicts an example of OTIS swapped network $O R_{5}$. Now we calculate certain degree based topological indices of OTIS swapped network $O R_{k}$.

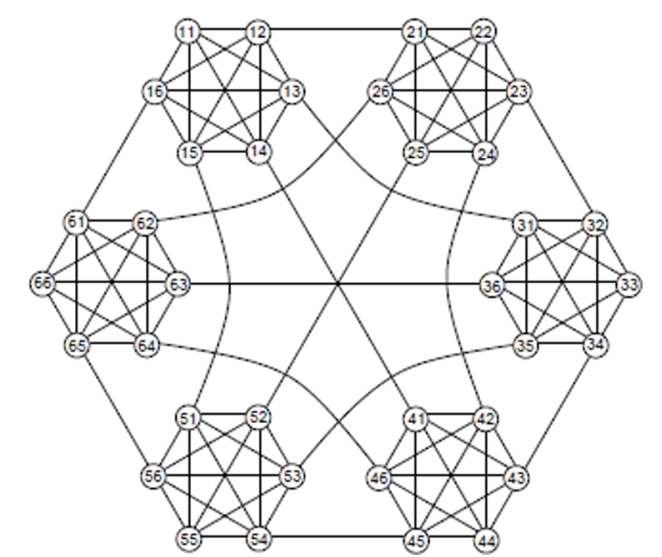

Figure 4.1: $O R_{5}$ OTIS swapped network

Theorem 4.2. Let $O R_{k}$ be the graph of OTIS swapped network. Then we have

1. $M_{1}^{-}\left(U R_{m}, x\right)=n m x^{2 k^{l}+\perp^{1}}+\frac{m^{2}\left(m+m_{1} \perp^{1}\right)-n(1,+2 m)}{2} x^{2 m+2}$.

2. $M_{2}\left(O R_{m}, x\right)=n m x^{k^{2}+k}+\frac{n^{2}(m+1)-n(1+2 m)}{2} x^{(m+1)^{2}}$.

3. $M_{3}\left(O R_{m}, x\right)=n k x+\frac{n^{2}(m+1)-n(1+2 m)}{2}$.

4. $M_{4}\left(O R_{m}, x\right)=n m x^{k^{2}+k}+\frac{n^{2}(m+1)-n(1+2 m)}{2} x^{2(m+1)^{2}}$.

5. $M_{5}\left(O R_{m}, x\right)=n k x^{2 k^{2}+3 k+1}+\frac{n^{2}(m+1)-n(1+2 m)}{2} x^{2(m+1)^{2}}$.

6. $M_{a, b}\left(O R_{m}, x\right)=n k x^{a m+b m+b}+\frac{n^{2}(m+1)-n(1+2 m)}{2} x^{(a+b)(m+1)}$.

7. $M_{a, b}^{\prime}\left(O R_{m}, x\right)=n m x^{(m+1)(m+1+b)}+\frac{n^{2}(m+1)-n(1+2 m)}{2} x^{(m+1+a)(m+1+b)}$. 
Proof. The edge set of $O R_{k}$ has following two partitions,

$$
\begin{gathered}
E_{1}\left(O R_{m}\right)=\left\{e=u v \epsilon E\left(O R_{m}\right) \mid d_{u}=k, d_{v}=k+1\right\}, \\
E_{2}\left(O R_{m}\right)=\left\{e=u v \epsilon E\left(O R_{m}\right) \mid d_{u}=k+1, d_{v}=k+1\right\},
\end{gathered}
$$

such that

$$
\begin{gathered}
\left|E_{1}\left(O R_{m}\right)\right|=n k \\
\left|E_{2}\left(O R_{m}\right)\right|=\frac{n^{2}(k+1)-n(1+2 k)}{2},
\end{gathered}
$$

1. Using the edge partition and definition 2.1, we have

$$
\begin{aligned}
M_{1}\left(O R_{m}, x\right) & =\sum_{u v \in E\left(O R_{m}\right)} x^{d_{u}+d_{v}} \\
& =\sum_{u v \epsilon E_{1}\left(O R_{m}\right)(G)} x^{2 m+1}+\sum_{u v \epsilon E_{2}\left(O R_{m}\right)}\left(O R_{m}\right) x^{2 m+2} \\
& =\left|E_{1}\left(O R_{m}\right)\right| x^{2 m+1}+\left|E_{2}\left(O R_{m}\right)\right| x^{2 m+2} \\
& =n m x^{2 m+1}+\frac{n^{2}(m+1)-n(1+2 m)}{2} x^{2 m+2} .
\end{aligned}
$$

2. Using the edge partition and definition 2.2, we have

$$
\begin{aligned}
M_{2}\left(O R_{m}, x\right) & =\sum_{u v \in E\left(O R_{m}\right)} x^{d_{u} \cdot d_{v}} \\
& =\sum_{u v \epsilon E_{1}\left(O R_{m}\right)(G)} x^{m^{2}+m}+\sum_{u v \in M_{2}\left(O R_{m}\right)}\left(O R_{m}\right) x^{(m+1)^{2}} \\
& =\left|E_{1}\left(O R_{m}\right)\right| x^{m^{2}+m}+\left|E_{2}\left(O R_{m}\right)\right| x^{(m+1)^{2}} \\
& =n m x^{m^{2}+m}+\frac{n^{2}(m+1)-n(1+2 m)}{2} x^{(m+1)^{2}}
\end{aligned}
$$

3. Using the edge partition and definition 2.3 , we have

$$
\begin{aligned}
M_{3}\left(O R_{m}, x\right) & =\sum_{u v \in E\left(O R_{m}\right)} x^{\left|d_{u}-d_{v}\right|} \\
& =\sum_{u v \epsilon E_{1}\left(O R_{m}\right)(G)} x+\sum_{u v \epsilon E_{2}\left(O R_{m}\right)} x^{0} \\
& =\left|E_{1}\left(O R_{m}\right)\right| x^{1}+\left|E_{2}\left(O R_{m}\right)\right| x^{1} \\
& =n m x+\frac{n^{2}(m+1)-n(1+2 m)}{2} .
\end{aligned}
$$


4. Using the edge partition and definition 2.4, we have

$$
\begin{aligned}
M_{4}\left(O R_{m}, x\right) & =\sum_{u v \in E\left(O R_{m}\right)} x^{d_{u}\left(\left|d_{u}+d_{v}\right|\right)} \\
& =\sum_{u v \epsilon E_{1}\left(O R_{m}\right)} x^{m(2 m+1)}+\sum_{u v \epsilon E_{2}\left(O R_{m}\right)} x^{(m+1)(2 m+2)} \\
& =\left|E_{1}\left(O R_{m}\right)\right| x^{m^{2}+m}+\left|E_{2}(G)\right| x^{2(m+1)^{2}} \\
& =n m x^{m^{2}+m}+\frac{n^{2}(m+1)-n(1+2 m)}{2} x^{2(m+1)^{2}} .
\end{aligned}
$$

5. Using the edge partition and definition 2.5, we have

$$
\begin{aligned}
M_{5}\left(O R_{m}, x\right) & =\sum_{u v \in E\left(O R_{m}\right)} x^{d_{v}\left(\left|d_{u}+d_{v}\right|\right)} \\
& =\sum_{u v \in E_{1}\left(O R_{m}\right)} x^{(m+1)(2 m+1)}+\sum_{u v \in E_{2}\left(O R_{m}\right)} x^{(m+1)(2 m+2)} \\
& =\left|E_{1}\left(O R_{m}\right)\right| x^{2 m^{2}+3 m+1}+\left|E_{2}\left(O R_{m}\right)\right| x^{2(m+1)^{2}} \\
& =n m x^{2 m^{2}+3 m+1}+\frac{n^{2}(m+1)-n(1+2 m)}{2} x^{2(m+1)^{2}} .
\end{aligned}
$$

6. Using the edge partition and definition 2.6, we have

$$
\begin{aligned}
M_{a, b}\left(O R_{m}, x\right) & =\sum_{u v \epsilon E\left(O R_{m}\right)} x^{\left(a d_{u}+b d_{v}\right)} \\
& =\sum_{u v \in E_{1}\left(O R_{m}\right)} x^{a m+b(m+1)}+\sum_{u v \epsilon E_{2}\left(O R_{m}\right)} x^{a(m+1)+b(m+1)} \\
& =\left|E_{1}\left(O R_{m}\right)\right| x^{a m+b m+b}+\left|E_{2}\left(O R_{m}\right)\right| x^{(a+b)(m+1)} \\
& =n m x^{a m+b m+b}+\frac{n^{2}(m+1)-n(1+2 m)}{2} x^{(a+b)(m+1)} .
\end{aligned}
$$

7. Using the edge partition and definition 2.7, we have

$$
\begin{aligned}
M_{a, b}^{\prime}\left(O R_{m}, x\right) & =\sum_{u v \in E\left(O R_{m}\right)} x^{\left(d_{u}+a\right)\left(d_{v}+b\right)} \\
& =\sum_{u v \in E_{1}\left(O R_{m}\right)} x^{(m+a)(m+1+b)}+\sum_{u v \in E_{2}\left(O R_{m}\right)} x^{(m+1+a)+(m+1+b)} \\
& =\left|E_{1}\left(O R_{m}\right)\right| x^{(m+a)(m+1+b)}+\left|E_{2}\left(O R_{m}\right)\right| x^{(m+1+a)(m+1+b)} \\
& =n m x^{(m+a)+(m+1+b)}+\frac{n^{2}(m+1)-n(1+2 m)}{2} x^{(m+1+a)(k+1+b)} .
\end{aligned}
$$


Theorem 4.3. Let $O R_{m}$ be the graph of OTIS swapped network. Then we have

1. $\operatorname{Re} Z G_{1}\left(O R_{m}\right)=n^{2}$.

2. $R e Z G_{2}\left(O R_{m}\right)=(n m) \frac{m^{2}+m}{2 m+1}-\left(\frac{n^{2}(m+1)-n(1+2 m)}{2}\right) \frac{m+1}{2}$.

3. $\operatorname{Re} Z G_{3}\left(O R_{m}\right)=(n m)\left(2 m^{3}+3 m^{2} m\right)+n^{2}(m+1)-n(1+2 m)(m+1)^{3}$.

\section{Proof.}

1. Using the edge partition given in Theorem 4.2 and definition 2.8 , we have

$$
\begin{aligned}
\operatorname{Re} Z G_{1}\left(O R_{m}\right) & =\sum_{u v \in E(G)} \frac{d_{u}+d_{v}}{d_{u} \cdot d_{v}} \\
& =\sum_{u v \in E_{1}\left(O R_{m}\right)} \frac{m+m+1}{m(m+1)}+\sum_{u v \in E_{2}\left(O R_{m}\right)} \frac{m+1+m+1}{(m+1)(m+1)} \\
& =\left|E_{1}\left(O R_{m}\right)\right| \frac{2 m+1}{m^{2}+m}+\left|E_{2}\left(O R_{m}\right)\right| \frac{2}{m+1} \\
& =n^{2} .
\end{aligned}
$$

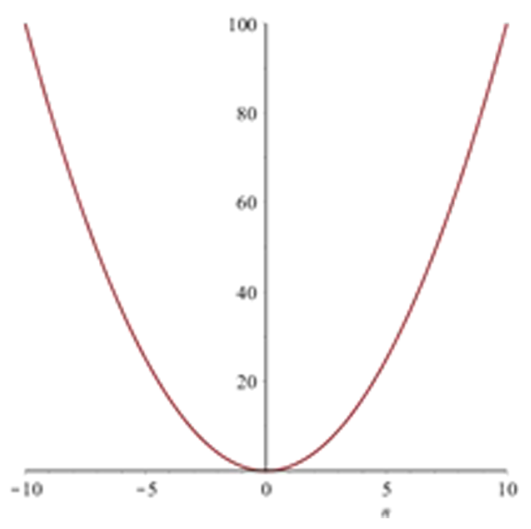

Figure 4.2: Plot of first redefined Zagreb index 
2. Using the edge partition given in Theorem 4.2 and definition 2.9, we have

$$
\begin{aligned}
R e Z G_{2}\left(O R_{m}\right) & =\sum_{u v \in E\left(O R_{m}\right)} \frac{d_{u} \cdot d_{v}}{d_{u}+d_{v}} \\
& =\sum_{u v \in E_{1}\left(O R_{m}\right)} \frac{m(m+1)}{m+m+1}+\sum_{u v \in E_{2}\left(O R_{m}\right)} \frac{(m+1)(m+1)}{m+1+m+1} \\
& =\left|E_{1}\left(O R_{m}\right)\right| \frac{m^{2}+m}{2 m+1}+\left|E_{2}\left(O R_{m}\right)\right| \frac{m+1}{2} \\
& =n m \cdot \frac{m^{2}+m}{2 m+1}-\left(\frac{n^{2}(m+1)-n(1+2 m)}{2}\right) \frac{m+1}{2} .
\end{aligned}
$$
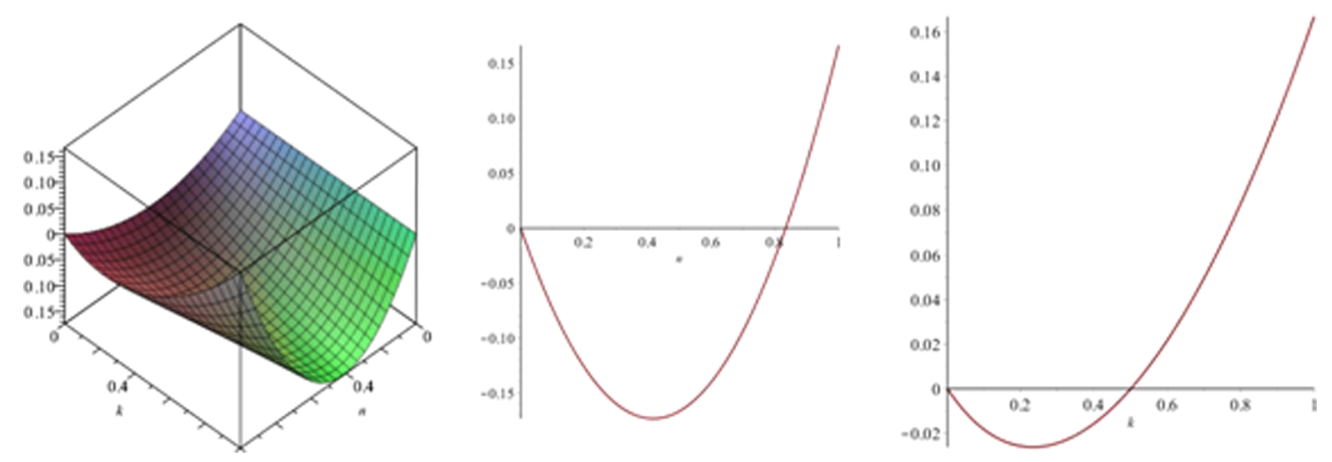

Figure 4.3: Plot of second redefined Zagreb index ( $3 \mathrm{D}$ (left), for $\mathrm{k}=1$ (middle), for $\mathrm{n}=1$ (right)

3. Using the edge partition given in Theorem 4.2 and definition 2.10, we have

$$
\begin{aligned}
\operatorname{Re} Z G_{3}\left(O R_{m}\right)= & \sum_{u v \in E\left(O R_{m}\right)}\left(d_{u} \cdot d_{v}\right)\left(d_{u}+d_{v}\right) \\
= & \sum_{u v \in E_{1}\left(O R_{m}\right)}(m(m+1)(m+m+1) \\
& +\sum_{u v \in E_{2}\left(O R_{m}\right)}((m+1) \cdot(m+1))(m+1+m+1)
\end{aligned}
$$




$$
\begin{aligned}
& =\left|E_{1}\left(O R_{m}\right)\right|\left(2 m^{3}+3 m^{2} m\right)+2\left|E_{2}\left(O R_{m}\right)\right|(m+1)^{3} \\
& =n m \cdot\left(2 m^{3}+3 m^{2} m\right)+n^{2}(m+1)-n(1+2 m)(m+1)^{3} .
\end{aligned}
$$
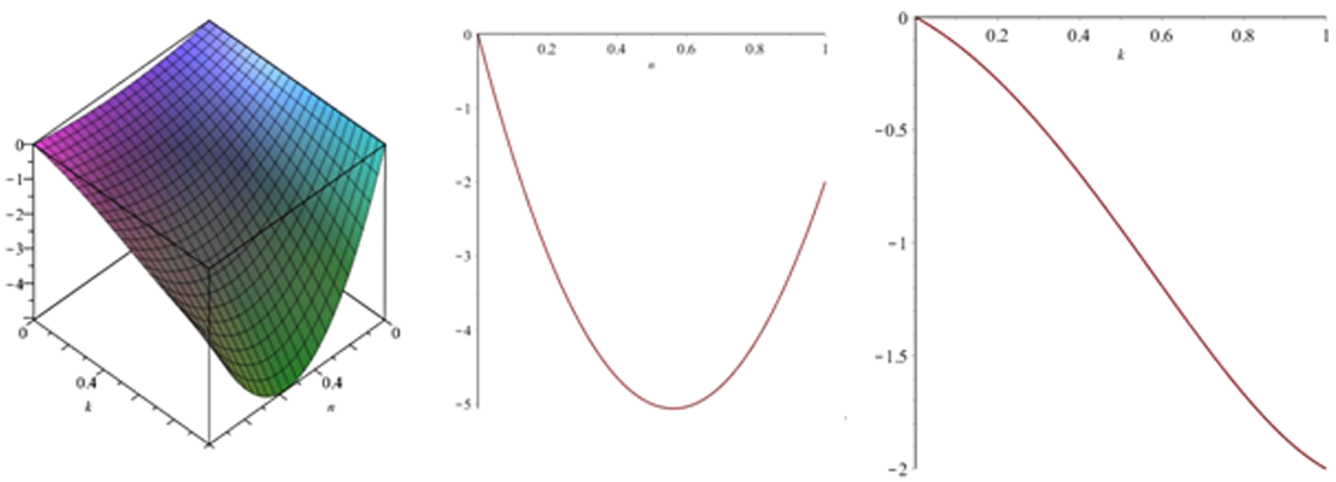

Figure 4.4: Plot of third redefined Zagreb index ( 3D (left), for $\mathrm{k}=1$ (middle), for $\mathrm{n}=1$ (right)

\subsection{Topological induces of $O P_{n}$ OTIS swapped Networks}

Let $P_{n}$ be path on $\mathrm{n}$ vertices and $O P_{n}$ be OTIS swapped network with basis network $P_{n}$. An OTIS swapped network with the basis network $O P_{6}$ is shown in Figure 5.

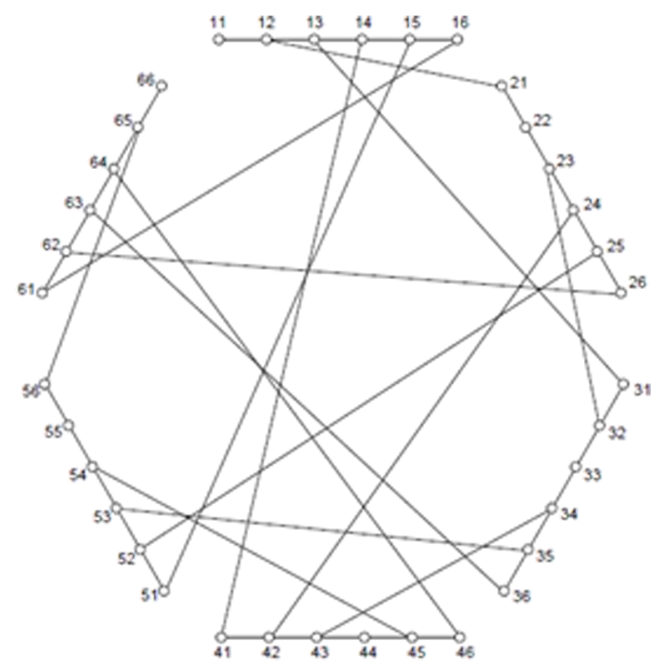

Figure 4.5: $O P_{6}$ OTIS swapped network 
Theorem 4.5. Let $O P_{n}$ be the graph of OTIS swapped network. Then we have

1. $M_{1}\left(O P_{n}, x\right)=5 x^{4}+(6 n-14) x^{5}+\frac{3(n-2)(n-3)}{2} x^{6}$.

2. $M_{2}\left(O P_{n}, x\right)=2 x^{3}+3 x^{4}+(6 n-14) x^{6}+\frac{3(n-2)(n-3)}{2} x^{9}$.

3. $M_{3}\left(O P_{n}, x\right)=2 x^{2}+(6 n-14) x+\frac{9(n-2)(n-3)}{2} x^{6}$.

4. $M_{4}\left(O P_{n}, x\right)=2 x^{4}+3 x^{8}+(6 n-14) x^{1} 0+\frac{3(n-2)(n-3)}{2} x^{18}$.

5. $M_{5}\left(O P_{n}, x\right)=3 x^{8}+(6 n-12) x^{1} 2+\frac{3(n-2)(n-3)}{2} x^{18}$.

6. $M_{a, b}\left(O P_{n}, x\right)=2 x^{a+3 b}+3 x^{2 a+2 b}+(6 n-14) x^{2 a+3 b}+\frac{3(n-2)(n-3)}{2} x^{3 a+3 b}$.

7. $M_{a, b}^{\prime}\left(O P_{n}, x\right)=2 x^{(1+a)(3+b)}+3 x^{(2+a)(2+b)}+(6 n-14) x^{(2+a)(3+b)}$ $+\frac{3(n-2)(n-3)}{2} x^{(3+a)(3+b)}$.

Proof. The edge set of $O P_{n}$ has following two partitions,

$$
\begin{aligned}
& E_{1\left(O P_{n}\right)}=\left[e=u v \epsilon E\left(O P_{n}\right) \mid d_{u}=1, d_{v}=3\right], \\
& E_{2\left(O P_{n}\right)}=\left[e=u v \epsilon E\left(O P_{n}\right) \mid d_{u}=2, d_{v}=2\right], \\
& E_{3\left(O P_{n}\right)}=\left[e=u v \epsilon E\left(O P_{n}\right) \mid d_{u}=2, d_{v}=3\right], \\
& E_{4\left(O P_{n}\right)}=\left[e=u v \epsilon E\left(O P_{n}\right) \mid d_{u}=3, d_{v}=3\right],
\end{aligned}
$$

such that

$$
\begin{gathered}
\left|E_{1}\left(O P_{n}\right)\right|=2, \\
\left|E_{2}\left(O P_{n}\right)\right|=3, \\
\left|E_{3}\left(O P_{n}\right)\right|=6 n-14, \\
\left|E_{4}\left(O P_{n}\right)\right|=\frac{3(n-2)(n-3)}{2},
\end{gathered}
$$

1. Using the edge partition and definition 2.1, we have

$$
\begin{aligned}
M_{1}\left(O P_{n}, x\right) & =\sum_{u v \epsilon E\left(O P_{n}\right)} x^{d_{u}+d_{v}} \\
& =\sum_{u v \epsilon E_{1}\left(O P_{n}\right)(G)} x^{4}+\sum_{u v \epsilon E_{2}\left(O P_{n}\right)} x^{4}+\sum_{u v \epsilon E_{3}\left(O P_{n}\right)} x^{5}+\sum_{u v \epsilon E_{4}\left(O P_{n}\right)} x^{6} \\
& =\left|E_{1}\left(O P_{n}\right)\right| x^{4}+\left|E_{2}\left(O P_{n}\right)\right| x^{4}+\left|E_{3}\left(O P_{n}\right)\right| x^{5}+\left|E_{4}\left(O P_{n}\right)\right| x^{6} \\
& =5 x^{4}+(6 n-14) x^{5}+\frac{3(n-2)(n-3)}{2} x^{6} .
\end{aligned}
$$


2. Using the edge partition and definition 2.2 , we have

$$
\begin{aligned}
M_{2}\left(O P_{n}, x\right) & =\sum_{u v \epsilon E\left(O P_{n}\right)} x^{d_{u} \cdot d_{v}} \\
& =\sum_{u v \in E_{1}\left(O P_{n}\right)\left(O P_{n}\right)} x^{3}+\sum_{u v \epsilon E_{2}\left(O P_{n}\right)} x^{4}+\sum_{u v \epsilon E_{3}\left(O P_{n}\right)} x^{6}+\sum_{u v \in E_{4}\left(O P_{n}\right)} x^{9} \\
& =\left|E_{1}\left(O P_{n}\right)\right| x^{3}+\left|E_{2}\left(O P_{n}\right)\right| x^{4}+\left|E_{3}\left(O P_{n}\right)\right| x^{6}+\left|E_{4}\left(O P_{n}\right)\right| x^{9} \\
& =2 x^{3}+3 x^{4}+(6 n-14) x^{6}+\frac{3(n-2)(n-3)}{2} x^{9} .
\end{aligned}
$$

3. Using the edge partition and definition 2.3 , we have

$$
\begin{aligned}
M_{3}\left(O P_{n}, x\right) & =\sum_{u v \epsilon E\left(O P_{n}\right)} x^{\left|d_{u}-d_{v}\right|} \\
& =\sum_{u v \epsilon E_{1}\left(O P_{n}\right)} x^{2}+\sum_{u v \in E_{2}\left(O P_{n}\right)} x^{0}+\sum_{u v \in E_{3}\left(O P_{n}\right)} x^{1}+\sum_{u v \in E_{4}\left(O P_{n}\right)} x^{0} \\
& =\left|E_{1}\left(O P_{n}\right)\right| x^{2}+\left|E_{2}\left(O P_{n}\right)\right|+\left|E_{3}\left(O P_{n}\right)\right| x+\left|E_{4}\left(O P_{n}\right)\right| \\
& =2 x^{2}+(6 n-14) x+\frac{9(n-2)(n-3)}{2} .
\end{aligned}
$$

4. Using the edge partition and definition 2.4, we have

$$
\begin{aligned}
M_{4}\left(O P_{n}, x\right) & =\sum_{u v \epsilon E\left(O P_{n}\right)} x^{d_{u}\left(\left|d_{u}+d_{v}\right|\right)} \\
& =\sum_{u v \epsilon E_{1}\left(O P_{n}\right)} x^{4}+\sum_{u v \epsilon E_{2}\left(O P_{n}\right)} x^{8}+\sum_{u v \epsilon E_{3}\left(O P_{n}\right)} x^{10}+\sum_{u v \epsilon E_{4}\left(O P_{n}\right)} x^{18} \\
& =\left|E_{1}\left(O P_{n}\right)\right| x^{4}+\left|E_{2}\left(O P_{n}\right)\right| x^{8}+\left|E_{3}\left(O P_{n}\right)\right| x^{10}+\left|E_{4}\left(O P_{n}\right)\right| x^{18} \\
& =2 x^{4}+3 x^{8}+(6 n-14) x^{10}+\frac{3(n-2)(n-3)}{2} x^{18} .
\end{aligned}
$$

5. Using the edge partition and definition 2.5, we have

$$
\begin{aligned}
M_{5}\left(O P_{n}, x\right) & =\sum_{u v \epsilon E\left(O P_{n}\right)} x^{d_{v}\left(\left|d_{u}+d_{v}\right|\right)} \\
& =\sum_{u v \epsilon E_{1}\left(O P_{n}\right)} x^{12}+\sum_{u v \epsilon E_{2}\left(O P_{n}\right)} x^{8}+\sum_{u v \epsilon E_{3}\left(O P_{n}\right)} x^{15}+\sum_{u v \epsilon E_{4}\left(O P_{n}\right)} x^{18} \\
& =\left|E_{1}\left(O P_{n}\right)\right| x^{12}+\left|E_{2}\left(O P_{n}\right)\right| x^{8}+\left|E_{3}\left(O P_{n}\right)\right| x^{15}+\left|E_{4}\left(O P_{n}\right)\right| x^{18} \\
& =2 x^{12}+3 x^{8}+(6 n-14) x^{15}+\frac{3(n-2)(n-3)}{2} x^{18} .
\end{aligned}
$$


6. Using the edge partition and definition 2.6, we have

$$
\begin{aligned}
M_{(a, b)}\left(O P_{n}, x\right)= & \sum_{u v \epsilon E\left(O P_{n}\right)} x^{\left(a d_{u}+b d_{v}\right)} \\
= & \sum_{u v \epsilon E_{1}\left(O P_{n}\right)} x^{a+3 b}+\sum_{u v \epsilon E_{2}\left(O P_{n}\right)} x^{2 a+2 b} \\
& +\sum_{u v \epsilon E_{3}\left(O P_{n}\right)} x^{2 a+3 b}+\sum_{u v \epsilon E_{4}\left(O P_{n}\right)} x^{3 a+3 b} \\
= & \left|E_{1}\left(O P_{n}\right)\right| x^{a+3 b}+\left|E_{2}\left(O P_{n}\right)\right| x^{2 a+2 b} \\
& +\left|E_{3}\left(O P_{n}\right)\right| x^{2 a+3 b}+\left|E_{4}\left(O P_{n}\right)\right| x^{3 a+3 b} \\
= & 2 x^{a+3 b}+3 x^{2 a+2 b}+(6 n-14) x^{2 a+3 b}+\frac{3(n-2)(n-3)}{2} x^{3 a+3 b} .
\end{aligned}
$$

7. Using the edge partition and definition 2.7, we have

$$
\begin{aligned}
M_{(a, b)}^{\prime}\left(O P_{n}, x\right)= & \sum_{u v \in E\left(O P_{n}\right)} x^{\left(d_{u}+a\right)\left(d_{v}+b\right)} \\
= & \sum_{u v \in E_{1}\left(O P_{n}\right)} x^{(1+a)(3+b)}+\sum_{u v \epsilon E_{2}\left(O P_{n}\right)} x^{(2+a)(2+b)} \\
& +\sum_{u v \epsilon E_{3}\left(O P_{n}\right)} x^{(2+a)(3+b)}+\sum_{u v \epsilon E_{4}\left(O P_{n}\right)} x^{(3+a)(3+b)} \\
= & \left|E_{1}\left(O P_{n}\right)\right| x^{(1+a)(3+b)}+\left|E_{2}\left(O P_{n}\right)\right| x^{(2+a)(2+b)} \\
& +\left|E_{3}\left(O P_{n}\right)\right| x^{(2+a)(3+b)}+\left|E_{4}\left(O P_{n}\right)\right| x^{(3+a)(3+b)} \\
= & 2 x^{(1+a)(3+b)}+3 x^{(2+a)(2+b)}+(6 n-14) x^{(2+a)(3+b)} \\
& +\frac{3(n-2)(n-3)}{2} x^{(3+a)(3+b)} .
\end{aligned}
$$

Theorem 4.6. Let $O P_{n}$ be the graph of OTIS swapped network. Then we have

1. $\operatorname{Re} Z G_{1}\left(O P_{n}\right)=n^{2}$.

2. $R e Z G_{2}\left(O P_{n}\right)=\frac{6}{5}-\frac{81}{20} n+\frac{9}{4} n^{2}$.

3. $\operatorname{Re} Z G_{3}\left(O P_{n}\right)=78 n^{2}-210 n+120$. 


\section{Proof.}

1. Using the edge partition given in Theorem 4.5 and definition 2.8 , we have

$$
\begin{aligned}
R e Z G_{1}\left(O P_{n}\right) & =\sum_{u v \in E\left(O P_{n}\right)} \frac{d_{u}+d_{v}}{d_{u} \cdot d_{v}} \\
& =\sum_{u v \in E_{1}\left(O P_{n}\right)} \frac{4}{3}+\sum_{u v \in E_{2}\left(O P_{n}\right)} \frac{4}{4}+\sum_{u v \in E_{3}\left(O P_{n}\right)} \frac{5}{6}+\sum_{u v \in E_{4}\left(O P_{n}\right)} \frac{6}{9} \\
& =\left|E_{1}\left(O P_{n}\right)\right| \frac{4}{3}+\left|E_{2}\left(O P_{n}\right)\right|+\left|E_{3}\left(O P_{n}\right)\right| \frac{5}{6}+\left|E_{4}\left(O P_{n}\right)\right| \frac{2}{3} \\
& =n^{2} .
\end{aligned}
$$

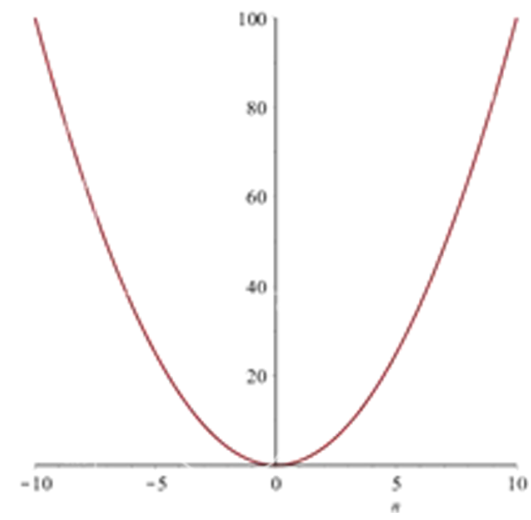

Figure 4.6: Plot of first redefined Zagreb index

2. Using the edge partition given in Theorem 4.5 and definition 2.9, we have

$$
\begin{aligned}
R e Z G_{2}\left(O P_{n}\right) & =\sum_{u v \in E\left(O P_{n}\right)} \frac{d_{u} \cdot d_{v}}{d_{u}+d_{v}} \\
& =\sum_{u v \in E_{1}\left(O P_{n}\right)} \frac{3}{4}+\sum_{u v \epsilon E_{2}\left(O P_{n}\right)} \frac{4}{4}+\sum_{u v \in E_{3}\left(O P_{n}\right)} \frac{6}{5}+\sum_{u v \epsilon E_{4}\left(O P_{n}\right)} \frac{9}{6} \\
& =\left|E_{1}\left(O P_{n}\right)\right| \frac{3}{4}+\left|E_{2}\left(O P_{n}\right)\right|+\left|E_{3}\left(O P_{n}\right)\right| \frac{6}{5}+\left|E_{4}\left(O P_{n}\right)\right| \frac{3}{2} \\
& =\frac{6}{5}-\frac{81}{20} n+\frac{9}{4} n^{2} .
\end{aligned}
$$




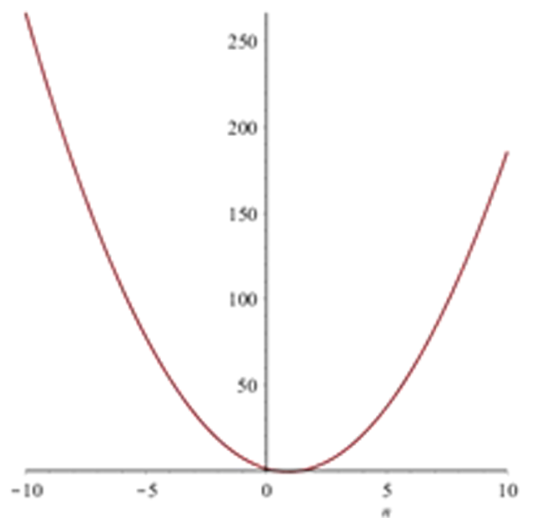

Figure 4.6: Plot of second redehned 'Lagreb index

3. Using the edge partition given in Theorem 4.5 and definition 2.10, we have

$$
\begin{aligned}
\operatorname{Re} Z G_{3}\left(O P_{n}\right) & =\sum_{u v \epsilon E\left(O P_{n}\right)}\left(d_{u} \cdot d_{v}\right)\left(d_{u}+d_{v}\right) \\
& =\sum_{u v \epsilon E_{1}\left(O P_{n}\right)} 12+\sum_{u v \epsilon E_{2}\left(O P_{n}\right)} 16+\sum_{u v \epsilon E_{3}\left(O P_{n}\right)} 30+\sum_{u v \epsilon E_{4}\left(O P_{n}\right)} 54 \\
& =\left|E_{1}\left(O P_{n}\right)\right| 12+\left|E_{2}\left(O P_{n}\right)\right| 16+\left|E_{3}\left(O P_{n}\right)\right| 30+\left|E_{4}\left(O P_{n}\right)\right| 54 \\
& =78 n^{2}-210 n+120 .
\end{aligned}
$$

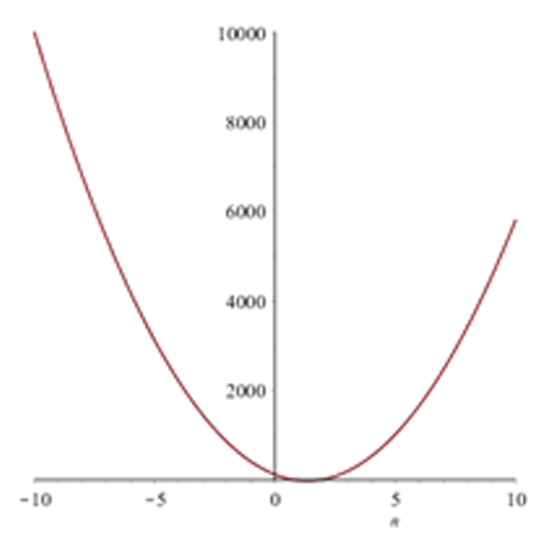

Figure 4.8: Plot of third redefined Zagreb index 


\section{Conclusion}

It is important to calculate topological indices of networks, because it is proved fact that topological indices help to predict many properties without going to the wet lab. There are more than 148 topological indices but none of them can completely describe all properties of a chemical compound. Therefore there is always room to define and study new topological indices. Redefined Zagreb indices are one step in this direction and are very close to Zagreb indices. Zagreb indices are very well studied by chemists and mathematician due to its huge applications in chemistry. It is an interesting problem for researchers to study chemical properties and bonds of redefined Zagreb indices.

\section{References}

[1] M. Ajmal,W. Nazeer, M. Munir,S. M. Kang and Y. C. Kwun, "M-polynomials and topological indices of generalized prism and toroidal polyhex networks", Symmetry, Under review.

[2] A. Ali, W. Nazeer, M. Munir, and S. M. Kang, "M-Polynomials and topological indices of zigzag and rhombic benzenoid systems", Open chemistry, vol. 16, no. 1, pp. 73-78, Feb. 2018, doi: 10.1515/chem-2018-0010

[3] U. Ali, Y. Ahmad, and M. S. Sardar, "On 3-total edge product cordial labeling of tadpole, book and flower graphs", Open journal of mathematical sciences, vol. 4, pp. 48-55, Mar. 2020, doi: 10.30538/oms2020.0093

[4] D. Amić, D. Bešlo, B. Lučić, S. Nikolić, and N. Trinajstić, "The vertexconnectivity index revisited", Journal of chemical information and computer sciences, vol. 38, no. 5, pp. 819-822, Sep. 1998, doi: $10.1021 / \mathrm{ci} 980039 \mathrm{~b}$

[5] F. Asif, Z. Zahid and S. Zafar, "Leap Zagreb and leap hyper-Zagreb indices of Jahangir and Jahangir derived graphs", Engineering and applied science letter, vol. 3, no. 2, pp. 1-8, Apr. 2020. [On line]. Available: https://bit.ly/2UOwvo2

[6] A. R. Bindusree, N. Cangul, V. Lokesha, and S. Cevik, "Zagreb polynomials of three graph operators", Filomat, vol. 30, no. 7, pp. 1979-1986, 2016, doi: 10.2298/FIL1607979B 
[7] B. Bollobas and P. Erdos, "Graphs of extremal weights", Ars combinatoria, vol. 50, pp. 225-233, 1998.

[8] E. Deutsch and Klavžar, "S. M-Polynomial, and degree-based topological indices", Iranian journal of mathematical chemistry, vol. 6, no. 2, pp. 93-102, 2015, doi: 10.22052/IJMC.2015.10106

[9] G. H. Fath-Tabar. "Zagreb polynomial and Pi indices of some nano structures", Digest journal of nanomaterials and biostructures, vol. 4, no,1, pp. 189-191, Mar. 2009. [On line]. Available: https://bit.ly/3d5Dv6h

[10] G. H. Fath-Tabar, "Old and new Zagreb index", MATCH communications in mathematical and in computer chemistry, vol. 65, no. 1, pp. 79-84, 2011. [On line]. Available: https://bit.ly/2N6LcyK

[11] W. Gao, W. Wang, and M. R. Farahani, "Topological indices study of molecular structure in anticancer drugs", Journal of chemistry, vol. 2016, Art ID. 3216327, 2016, doi: 10.1155/2016/3216327

[12] W. Gao, M. Younas, A. Farooq, A. Virk, and W. Nazeer, "Some reverse degree-based topological indices and polynomials of dendrimers", Mathematics, vol. 6, no. 10, Art ID 214, Oct. 2018, doi: $10.3390 /$ math6100214

[13] I. Gutman, "Some properties of the Wiener polynomials", Graph theory notes New York, vol.125, pp. 13-18, 1993.

[14] I. Gutman and K. C. Das, "The first Zagreb indices 30 years after", MATCH communications in mathematical and in computer chemistry, vol. 50, pp. 83-92, 2004. [On line]. Available: https://bit.ly/3ea6XJF

[15] J. Liu, M. Younas, M. Habib, M. Yousaf and W. Nazeer, "M-Polynomials and degree-based topological indices of $\mathrm{VC}_{5} \mathrm{C}_{7}[\mathrm{p}, \mathrm{q}]$ and $\mathrm{HC}_{5} \mathrm{C}_{7}[\mathrm{p}, \mathrm{q}]$ nanotubes", IEEE Access, vol. 7, pp. 41125-41132, 2019, doi: 10.1109/ACCESS.2019.2907667

[16] M. Munir, W. Nazeer, S. Rafique, and S. Kang, "M-Polynomial and related topological indices of nanostar dendrimers", Symmetry, vol. 8, no. 9, Art ID. 97, Sep. 2016, doi: 10.3390/sym8090097 
[17] M. Munir, W. Nazeer, A. Nizami, S. Rafique, and S. Kang, "M-Polynomials and topological indices of titania nanotubes", Symmetry, vol. 8, no. 11, Art ID. 117, Oct. 2016, doi: 10.3390/sym8110117

[18] M. Munir, W. Nazeer, Z. Shahzadi, and S. Kang, "Some invariants of circulant graphs", Symmetry, vol. 8, no. 11, Art ID. 134, Nov. 2016, doi: $10.3390 /$ sym8110134

[19] M. Munir, W. Nazeer, S. Rafique, and S. Kang, "M-Polynomial and degree-based topological indices of polyhex nanotubes", Symmetry, vol. 8, no. 12, Art ID. 149, Dec. 2016, doi: 10.3390/sym8120149

[20] M. Randic, "Characterization of molecular branching", Journal of the American Chemical Society, vol. 97, no. 23, pp. 6609-6615, Nov. 1975, doi: $10.1021 / \mathrm{ja} 00856 \mathrm{a} 001$

[21] P. S. Ranjini, V. Lokesha, A. R. Bindusree, and M. P. Raju, "New bounds on Zagreb indices and the Zagreb co-indices", Boletim da Sociedade Paranaense de Matemática, vol. 31, no. 1, pp. 51-55, 2013, doi: 10.5269/bspm.v31i1.15272

[22] P. S. Ranjini ,V. Lokesha and A. Usha, "Relation between phenylene and hexagonal squeeze using harmonic index", International journal of graph theory, vol. 1, no. 4, pp. 116-121, 2013.

[23] A. Shah and S. A. S. Bokhary, "On chromatic polynomial of certain families of dendrimer graphs", Open journal of mathematical sciences, vol. 3, pp. 404-416, Dec. 2019, doi:10.30538/oms2019.0083

[24] A. Tabassum, M. A. Umar, M. Perveen, and A. Raheem, "Antimagicness of subdivided fans", Open journal of mathematical sciences, vol. 4, no. 1, pp. 18-22, Feb. 2020, doi: 10.30538/oms2020.0089

[25] M. A. Umar, N. Ali, A. Tabassum and B. R. Ali, "Book graphs are cycle antimagic", Open journal of mathematical sciences, vol. 3, pp. 184-90, Jun. 2019, doi: 10.30538/oms2019.0061

[26] H. Wiener, "Structural determination of paraffin boiling points", Journal of the American Chemical Society, vol. 69, no. 1, pp. 17-20, Jan. 1947, doi: 10.1021/ja01193a005 